\title{
ATTRACTORS OF ITERATED FUNCTION SYSTEMS AND ASSOCIATED GRAPHS
}

\author{
Dan Dumitru and AleXandru Mihail
}

\begin{abstract}
The aim of this article is to establish some conditions under which the attractors of iterated function systems become dendrites. We associate to an attractor of an iterated function system (IFS) some graphs and we prove that for a large class of IFSs their attractors are dendrites if the associated graphs are trees. We also give some examples of such sets.
\end{abstract}

\section{Introduction}

We start by a brief presentation of iterated function systems, IFSs for short. Iterated function systems were conceived in the present form by John Hutchinson in [6], popularized by Michael Barnsley in [2] and are one of the most common and general ways to generate fractals. Many of the important examples of functions and sets with special and unusual properties turn out to be fractal sets or functions whose graphs are fractal sets and a great part of them are attractors of IFSs. There is a current effort to extend the classical Hutchinson's framework to more general spaces and infinite iterated function systems (IIFSs) or, more generally, to multifunction systems and to study them ([1], [8-15], [17-19]). A recent such example can be found in [9], where the Lipscomb's space, which is an important example in dimension theory, can be obtain as an attractor of an IIFS defined in a very general settings. Although the fractal sets are defined with measure theory, being sets with noninteger Hausdorff dimension ([4], [5]), it turns out that they have interesting topological properties (see [7], [14], [20]). The topological properties of fractal sets have a great importance in analysis on fractals as we can see in [7]. One of the most important results in this direction, which states when the attractor of an IFS is a connected set, is given in [7] or [20]. Other results on this problem can be found in [3].

In this article we intend to find sufficient conditions for an attractor of an IFS to be a dendrite. These conditions are necessary for a large class of IFSs. The paper is divided in four parts. The first part is the introduction. In the

2003 Mathematics Subject Classification. 28A80.

Key words and phrases. attractors, iterated function system, dendrites.

Received December 4, 2013. 
second part is given the description of the shift space of an iterated function system. The main result, theorem 3.1, is contained in the third part. The last part contains some examples: the Hata's tree-like set, the Cross set.

For a metric space $(X, d)$, we denote by $\mathscr{K}(X)$ the set of nonempty compact subsets of $X$. For a set $A \subset X$ we denote by $d(A)$ the diameter of $A$, that is $d(A)=\sup _{x, y \in A} d(x, y)$.

Definition 1.1. Let $(X, d)$ be a metric space. The application $h: \mathscr{K}(X) \times$ $\mathscr{K}(X) \rightarrow[0,+\infty)$ defined by $h(A, B)=\max (d(A, B), d(B, A))$, where $d(A, B)=$ $\sup _{x \in A} d(x, B)=\sup _{x \in A}\left(\inf _{y \in B} d(x, y)\right)$ is called the Hausdorff-Pompeiu metric.

Remark 1.1 ([1], [2], [9], [16]). $(\mathscr{K}(X), h)$ is a complete metric space if $(X, d)$ is a complete metric space, compact if $(X, d)$ is compact and separable if $(X, d)$ is separable.

Definition 1.2. Let $(X, d)$ be a metric space. For a function $f: X \rightarrow X$ let us denote by $\operatorname{Lip}(f) \in[0,+\infty]$ the Lipschitz constant associated to $f$, which is $\operatorname{Lip}(f)=\sup _{x, y \in X ; x \neq y} \frac{d(f(x), f(y))}{d(x, y)}$.

We say that $f$ is a Lipschitz function if $\operatorname{Lip}(f)<+\infty$ and a contraction if $\operatorname{Lip}(f)<1$.

DEFINITION 1.3. An iterated function system $(I F S)$ on a metric space $(X, d)$ consists in a finite family of contractions $\left(f_{k}\right)_{k=\overline{1, n}}$ on $X$ and it is denoted by $\mathscr{S}=\left(X,\left(f_{k}\right)_{k=\overline{1, n}}\right)$.

DeFINITION 1.4. For an IFS, $\mathscr{S}=\left(X,\left(f_{k}\right)_{k=\overline{1, n}}\right)$, the function $F_{\mathscr{S}}: \mathscr{K}(X)$ $\rightarrow \mathscr{K}(X)$ defined by $F_{\mathscr{S}}(B)=\bigcup_{k=1}^{n} f_{k}(B)$ is called the fractal operator associated with the IFS $\mathscr{S}$.

Remark 1.2 ([1], [2], [4], [5], [16]). The function $F_{\mathscr{S}}$ is a contraction satisfying $\operatorname{Lip}\left(F_{\mathscr{S}}\right) \leq \max _{k=\overline{1, n}} \operatorname{Lip}\left(f_{k}\right)$.

Using Banach's contraction theorem there exists, for an IFS $\mathscr{S}=$ $\left(X,\left(f_{k}\right)_{k=\overline{1, n}}\right)$, an unique set $A(\mathscr{S})$ such that $F_{\mathscr{S}}(A(\mathscr{S}))=A(\mathscr{S})$, which is called the attractor of the IFS $\mathscr{S}$. More precisely we have the following well-known result.

THEOREM 1.1 ([1], [2], [4], [5], [16]). Let $(X, d)$ be a complete metric space and $\mathscr{S}=\left(X,\left(f_{k}\right)_{k=\overline{1, n}}\right)$ an IFS with $c=\max _{k=\overline{1, n}} \operatorname{Lip}\left(f_{k}\right)<1$. Then there exists a unique set $A(\mathscr{S}) \in \mathscr{K}(X)$ such that $F_{\mathscr{S}}(A(\mathscr{S}))=A(\mathscr{S})$. Moreover, for any $H_{0} \in \mathscr{K}(X)$ the sequence $\left(H_{n}\right)_{n \geq 1}$ defined by $H_{n+1}=F_{\mathscr{S}}\left(H_{n}\right)$ is convergent to $A(\mathscr{S})$. For the speed of the convergence we have the following estimation $h\left(H_{n}, A(\mathscr{S})\right) \leq \frac{c^{n}}{1-c} h\left(H_{0}, H_{1}\right)$. 
Definition 1.5. 1) By a graph we understand a pair $(I, G)$, where $G$ is a subset of the set $\{\{i, j\} \mid i, j \in I$ and $i \neq j\}$.

2) A graph $(I, G)$ is called connected if for every $i, j \in I$ there exists $\left(i_{k}\right)_{k=\overline{1, n}} \subset I$ such that $i_{1}=i, i_{n}=j$ and $\left\{i_{k}, i_{k+1}\right\} \in G$ for every $k \in\{1,2, \ldots$, $n-1\}$.

3) Let $(I, G)$ be a graph. A family of vertices $\left(i_{1}, \ldots, i_{m}\right)$ is a cycle if $\left\{i_{k}, i_{k+1}\right\} \in G$ for every $k \in\{1, \ldots, n\}$ and $i_{k} \notin\left\{i_{k+1}, i_{k+2}\right\}$ for every $k \in\{1, \ldots, n\}$, where by $i_{m+1}$ we understand $i_{1}$, by $i_{m+2}$ we understand $i_{2}$ and so on.

4) A graph $(I, G)$ is called a tree if it is connected and has no cycles.

Remark 1.3. We remark that a cycle has at least 3 elements.

Definition 1.6. Let $X$ be a nonempty set and $\left(A_{i}\right)_{i \in I}$ a family of nonempty subsets of $X$. Then:

1) The graph $(I, G)$ where $G=\left\{\{i, j\} \mid i, j \in I\right.$ such that $A_{i} \cap A_{j} \neq \emptyset$ and $i \neq j\}$ is called the graph of the intersections associated with the family $\left(A_{i}\right)_{i \in I}$.

2) The family $\left(A_{i}\right)_{i \in I}$ is said to be connected if for every $i, j \in I$ there exists $\left(i_{k}\right)_{k=\overline{1, n}} \subset I$ such that $i_{1}=i, i_{n}=j$ and $A_{i_{k}} \cap A_{i_{k+1}} \neq \emptyset$ for every $k \in\{1,2, \ldots$, $n-1\}$. If a family $\left(A_{i}\right)_{i \in I}$ is not connected we say that it is disconnected. The family $\left(A_{i}\right)_{i \in I}$ is connected if and only if the graph $(I, G)$ is connected.

3) The family $\left(A_{i}\right)_{i \in I}$ is said to be a tree of sets if for every $i, j \in I$, such that $i \neq j$, there exists a unique sequence $\left(i_{k}\right)_{k=\overline{1 . n}} \subset I$, with $i_{1}, i_{2}, \ldots, i_{n}$ different, such that $i_{1}=i, i_{n}=j$, and $A_{i_{k}} \cap A_{i_{k+1}} \neq \emptyset$ for every $k \in\{1,2, \ldots, n-1\}$. The family $\left(A_{i}\right)_{i \in I}$ is a tree of sets if and only if the graph of the intersections of the family $\left(A_{i}\right)_{i \in I}$ is a tree.

4) On the family of sets $\left(A_{i}\right)_{i \in I}$ we consider the following equivalence relation: $A_{i} \sim A_{j}$ if and only if there exists $\left(i_{k}\right)_{k=\overline{1, n}} \subset I$ such that $i_{1}=i, i_{n}=j$ and $A_{i_{k}} \cap A_{i_{k+1}} \neq \emptyset$ for every $k \in\{1,2, \ldots, n-1\}$. A component of the family of sets $\left(A_{i}\right)_{i \in I}$ is a class of equivalence which corresponds to a connected subgraph of the graph of the intersections of the family $\left(A_{i}\right)_{i \in I}$.

Remark 1.4. 1) If the family of sets $\left(A_{i}\right)_{i \in I}$ is a tree of sets then the intersection of three different sets of the family is empty.

2) Let $\left(A_{i}\right)_{i \in I}$ be a family of sets, $X, Y$ be sets and $f: X \rightarrow Y$ be such that $\bigcup_{i \in I} A_{i} \subset X$. If the family of sets $\left(A_{i}\right)_{i \in I}$ is connected then the family of sets $\left(f\left(A_{i}\right)\right)_{i \in I}$ is connected. If the family of sets $\left(A_{i}\right)_{i \in I}$ is a tree and the function $f$ is injective then the family of sets $\left(f\left(A_{i}\right)\right)_{i \in I}$ is a tree.

3) Let $\left(A_{i}\right)_{i \in I}$ be a family of sets and $I_{1}, I_{2}, \ldots, I_{n}$ be a partition of the set $I$. We denote by $B_{k}$ the set $\bigcup_{i \in I_{k}} A_{i}$ for $k \in\{1,2, \ldots, n\}$ and by $C_{k}$ the set $\bigcup_{i, j \in I_{k} ; i \neq j}\left(A_{i} \cap A_{j}\right)$. If the family of sets $\left(A_{i}\right)_{i \in I_{1}},\left(A_{i}\right)_{i \in I_{2}}, \ldots,\left(A_{i}\right)_{i \in I_{3}}$ and $\left(B_{k}\right)_{k \in\{1,2, \ldots, n\}}$ are trees and $B_{k} \cap C_{k^{\prime}}=\emptyset$ for every $k, k^{\prime} \in\{1,2, \ldots, n\}$ different then the family of sets $\left(A_{i}\right)_{i \in I}$ is a tree.

Definition 1.7. A metric space $(X, d)$ is arcwise connected if for every $x, y \in X$ there exists a continuous function $\varphi:[0,1] \rightarrow X$ such that $\varphi(0)=x$ and 
$\varphi(1)=y$. A continuos function $\varphi$ as above is called a path between $x$ and $y$. We say that two continuous, injective functions $\varphi, \psi:[0,1] \rightarrow X$ are equivalent if there exists a function $u:[0,1] \rightarrow[0,1]$ continuous, bijective and increasing such that $\varphi \circ u=\psi$. A class of equivalence is named a curve. A curve is called injective if the functions from the definitions of the curve are injective.

Remark 1.5. We remark that two equivalent, continuous, injective functions have the same images.

Concerning the connectedness of the attractor of an IFS we have the following theorem.

THEOREM 1.2 ([7], [20]). Let $(X, d)$ be a complete metric space, $\mathscr{S}=$ $\left(X,\left(f_{k}\right)_{k=\overline{1, n}}\right)$ an IFS with $c=\max _{k=\overline{1, n}} \operatorname{Lip}\left(f_{k}\right)<1$ and $A(\mathscr{S})$ the attractor of $\mathscr{S}$. The following are equivalent:

1) The family $\left(A_{i}\right)_{i=\overline{1, n}}$ is connected, where $A_{i}=f_{i}(A(\mathscr{S}))$ for $k \in\{1, \ldots, n\}$.

2) $A(\mathscr{S})$ is arcwise connected.

3) $A(\mathscr{S})$ is connected.

\section{The shift space of an iterated function systems}

In this section we briefly present the shift space of an IFS. For more details one can see [2], [12] and [16]. We start with some set notations: $\mathbf{N}$ denotes the natural numbers, $\mathbf{N}^{*}=\mathbf{N}-\{0\}, \mathbf{N}_{n}^{*}=\{1,2, \ldots, n\}$. For two nonempty sets $A$ and $B, B^{A}$ denotes the set of functions from $A$ to $B$. By $\Lambda=\Lambda(B)$ we will understand the set $B^{\mathbf{N}^{*}}$ and by $\Lambda_{n}=\Lambda_{n}(B)$ we will understand the set $B^{\mathbf{N}_{n}^{*}}$. The elements of $\Lambda=\Lambda(B)=B^{\mathbf{N}^{*}}$ will be written as infinite words $\omega=\omega_{1} \omega_{2} \ldots$ $\omega_{m} \omega_{m+1} \cdots$, where $\omega_{m} \in B$ and the elements of $\Lambda_{n}=\Lambda_{n}(B)=B^{\mathbf{N}_{n}^{*}}$ will be written as finite words $\omega=\omega_{1} \omega_{2} \cdots \omega_{n}$. By $\lambda$ we will understand the empty word. Let us remark that $\Lambda_{0}(B)=\{\lambda\} . \quad \Lambda^{*}=\Lambda^{*}(B)$ is the set of all finite words; $\Lambda^{*}=\Lambda^{*}(B)=\bigcup_{n \geq 0} \Lambda_{n}(B)$. We denote by $|\omega|$ the length of the word $\omega$. An element of $\Lambda=\Lambda(B)$ is said to have length $+\infty$. If $\omega=\omega_{1} \omega_{2} \cdots \omega_{m} \omega_{m+1} \cdots$ or if $\omega=\omega_{1} \omega_{2} \cdots \omega_{n}$ and $n \geq m$ then $[\omega]_{m}:=\omega_{1} \omega_{2} \cdots \omega_{m}$. More generally if $l<m, \quad[\omega]_{m}^{l}=\omega_{l+1} \omega_{l+2} \cdots \omega_{m}$ and we have $[\omega]_{m}=[\omega]_{l}[\omega]_{m}^{l}$ for $\omega \in \Lambda_{n}(B)$ if $n \geq m>l \geq 1$ and for $\omega \in \Lambda(B)$ if $m>l \geq 1$. For two words $\alpha, \beta \in \Lambda^{*}(B) \cup$ $\Lambda(B), \alpha \prec \beta$ means $|\alpha| \leq|\beta|$ and $[\beta]_{|\alpha|}=\alpha$. For $\alpha \in \Lambda_{n}(B)$ and $\beta \in \Lambda_{m}(B)$ or $\beta \in \Lambda(B)$, by $\alpha \beta$ we will understand the joining of the words $\alpha$ and $\beta$ namely $\alpha \beta=\alpha_{1} \alpha_{2} \cdots \alpha_{n} \beta_{1} \beta_{2} \cdots \beta_{m}$ and respectively $\alpha \beta=\alpha_{1} \alpha_{2} \cdots \alpha_{n} \beta_{1} \beta_{2} \cdots \beta_{m} \beta_{m+1} \cdots$. On $\Lambda=\Lambda\left(\mathbf{N}_{n}^{*}\right)=\left(\mathbf{N}_{n}^{*}\right)^{\mathbf{N}^{*}}$ we consider the metric

$d_{s}(\alpha, \beta)=\sum_{k=1}^{\infty} \frac{1-\delta_{\alpha_{k}}^{\beta_{k}}}{3^{k}}$, where $\delta_{x}^{y}=\left\{\begin{array}{ll}1 & \text { if } x=y \\ 0 & \text { if } x \neq y\end{array}, \alpha=\alpha_{1} \alpha_{2} \cdots\right.$ and $\beta=\beta_{1} \beta_{2} \cdots$ 
Let $(X, d)$ be a complete metric space, $\mathscr{S}=\left(X,\left(f_{k}\right)_{k=1, n}\right)$ an IFS on $X$ and $A=A(\mathscr{S})$ the attractor of the IFS $\mathscr{S}$. For $\omega=\omega_{1} \omega_{2} \cdots \omega_{m} \in \Lambda_{m}\left(\mathbf{N}_{n}^{*}\right), f_{\omega}$ denotes $f_{\omega_{1}} \circ f_{\omega_{2}} \circ \cdots \circ f_{\omega_{m}}$ and $H_{\omega}$ denotes $f_{\omega}(H)$ for a set $H \subset X$. By $H_{\lambda}$ we will understand the set $H$. In particular $A_{\omega}=f_{\omega}(A)$.

The main results concerning the relation between the attractor of an IFS and the shift space is contained in the following theorem.

THEOREM 2.1 ([2], [11], [16]). Let $(X, d)$ be a complete metric space. If $A=A(\mathscr{S})$ is the attractor of the IFS $\mathscr{S}=\left(X,\left(f_{k}\right)_{k=\overline{1, n}}\right)$ then: precisely

1) For $\omega \in \Lambda=\Lambda\left(\mathbf{N}_{n}^{*}\right), A_{[\omega]_{m+1}} \subset A_{[\omega]_{m}}$ and $d\left(A_{[\omega]_{m}}\right) \rightarrow 0$ when $m \rightarrow \infty$; more

$$
d\left(A_{[\omega]_{m}}\right) \leq c^{m} d(A) .
$$

2) If $a_{\omega}$ is defined by $\left\{a_{\omega}\right\}=\bigcap_{m \geq 1} A_{[\omega]_{m}}$, then $d\left(e_{[\omega]_{m}}, a_{\omega}\right) \rightarrow 0$ when $m \rightarrow \infty$, where $e_{[\omega]_{m}}$ is the unique fixed point of $f_{[\omega]_{m}}$.

3) $A=A(\mathscr{S})=\bigcup_{\omega \in \Lambda}\left\{a_{\omega}\right\}, \quad A_{\alpha}=\bigcup_{\omega \in \Lambda}\left\{a_{\alpha \omega}\right\}$ for every $\alpha \in \Lambda^{*}, \quad A=$ $\bigcup_{\omega \in \Lambda_{m}} A_{\omega}$ for every $m \in \mathbf{N}^{*}$ and more general $A_{\alpha}=\bigcup_{\omega \in \Lambda_{m}} A_{\alpha \omega}$ for every $\alpha \in \Lambda^{*}$ and every $m \in \mathbf{N}^{*}$.

4) The set $\left\{e_{[\omega]_{m}} \mid \omega \in \Lambda\right.$ and $\left.m \in \mathbf{N}^{*}\right\}$ is dense in $A$.

5) The function $\pi: \Lambda \rightarrow A$ defined by $\pi(\omega)=a_{\omega}$ is continuous and surjective.

Definition 2.1. The function $\pi: \Lambda \rightarrow A=A(\mathscr{S})$ from the theorem 2.1. is called the canonical projection from the shift space on the attractor of the IFS $\mathscr{S}$.

\section{Main results}

The aim of this article is to establish sufficient conditions under which the attractor of an iterated function system becomes a dendrite.

We will start with some general properties of the dendrites.

Definition 3.1. The metric space $(X, d)$ is called a dendrite if for any $x, y \in X$ there exists an unique equivalence class of continuous, injective functions $\varphi:[0,1] \rightarrow X$ such that $\varphi(0)=x$ and $\varphi(1)=y$ (i.e. there exists an unique injective curve joining $x$ with $y$ ).

Lemma 3.1. Let $(X, d)$ be a dendrite and $B$ a subset of $X$. Then $B$ is a dendrite if and only if $B$ is arcwise connected.

Lemma 3.2. Let $(X, d)$ be a dendrite and $A_{1}, A_{2}, \ldots, A_{n}$ be subsets of $X$ such that $A_{1}, A_{2}, \ldots, A_{n}$ are also dendrites. Then $A_{1} \cap A_{2} \cap \cdots \cap A_{n}$ is a dendrite.

Proof. We denote the set $A_{1} \cap A_{2} \cap \cdots \cap A_{n}$ by $B$ and we consider $x, y \in B$. Thus $x, y \in A_{j}$, for every $j \in\{1, \ldots, n\}$. So for all $j \in\{1, \ldots, n\}$ there exist the continuous, injective functions $\varphi_{j}:[0,1] \rightarrow A_{i_{j}}$ such that $\varphi_{j}(0)=x$ and $\varphi_{j}(1)=y$. 
Since $X$ is a dendrite, $\varphi_{1}, \ldots, \varphi_{m}$ must be equivalent, which means that $\varphi_{1}([0,1])=\cdots=\varphi_{m}([0,1]) \subset B$. It follows that $B$ is arcwise connected and from lemma $3.1 B$ is a dendrite.

COROllary 3.1. Let $(X, d)$ be a complete metric space and $\mathscr{S}=$ $\left(X,\left(f_{k}\right)_{k=\overline{1, n}}\right)$ an IFS. We denote by $A$ the attractor of $\mathscr{S}$, by $A_{k}$ the set $f_{k}(A)$ for $k \in\{1, \ldots, n\}$. We suppose that $f_{k}$ is an injective function on $A$ for all $k \in\{1, \ldots, n\}$ and $A$ is a dendrite. Then $A_{i_{1}} \cap A_{i_{2}} \cap \cdots \cap A_{i_{m}}$ is a dendrite for all $i_{1}, \ldots, i_{m} \in\{1, \ldots, n\}$.

Proof. Let us suppose that $A$ is a dendrite. Since $f_{k}$ is injective on $A$ it follows that $A_{k}=f_{k}(A)$ is also a dendrite. We can apply lemma 3.2 to $\left(A, d_{\mid A}\right)$ and $A_{i_{1}}, A_{i_{2}}, \ldots, A_{i_{m}}$.

Notation 3.1. Let $a, b$ be real numbers such that $a<b$. $\mathscr{D}([a, b])$ denotes the set of the divisions of the interval $[a, b]$. For a division of the interval $[a, b]$,

$$
\Delta=\left(a=y_{0}<y_{1}<\cdots<y_{n}=b\right), \quad\|\Delta\|=\max _{k=\overline{0, n-1}}\left|y_{k}-y_{k+1}\right| .
$$

Lemma 3.3. Let $(X, d)$ be a metric space and $\varphi, \varphi^{\prime}:[0,1] \rightarrow X$ be continuous, injective functions such that there exist two sequences of divisions of the interval $[0,1],\left(\Delta_{l}\right)_{l \in \mathbf{N}} \in \mathscr{D}([0,1])$ and $\left(\Delta_{l}^{\prime}\right)_{l \in \mathbf{N}} \in \mathscr{D}([0,1])$ with the following properties:

a) $\Delta_{l}=\left(0=y_{0}^{l}<y_{1}^{l}<\cdots<y_{n_{l}}^{l}=1\right)$ and $\Delta_{l}^{\prime}=\left(0=z_{0}^{l}<z_{1}^{l}<\cdots<z_{n_{l}}^{l}=1\right)$ have the same number of elements for all $l \in \mathbf{N}$,

b) $\left\|\Delta_{l}\right\| \stackrel{l \rightarrow \infty}{\longrightarrow} 0$ and $\left\|\Delta_{l}^{\prime}\right\| \stackrel{l \rightarrow \infty}{\longrightarrow} 0$,

c) $\max _{k=\overline{0, n_{l}}} d\left(\varphi\left(y_{k}^{l}\right), \varphi^{\prime}\left(z_{k}^{l}\right)\right) \stackrel{l \rightarrow \infty}{\longrightarrow} 0$.

Then there exists an unique continuous, bijective and increasing function $u:[0,1] \rightarrow[0,1]$ such that $\varphi^{\prime} \circ u=\varphi$ (i.e. $\varphi$ and $\varphi^{\prime}$ are equivalent).

Proof. Let $t \in[0,1]$. Then there exists a sequence $\left(k_{l}(t)\right)_{l \in \mathbf{N}}$ of natural numbers such that $y_{k_{l}(t)}^{l} \leq t \leq y_{k_{l}(t)+1}^{l}$.

It is easy to see that $d\left(\varphi\left(y_{k_{l}(t)}^{l}\right), \varphi(t)\right) \stackrel{l \rightarrow \infty}{\longrightarrow} 0$. Therefore from point $\left.\mathrm{c}\right)$ $d\left(\varphi^{\prime}\left(z_{k_{l}(t)}^{l}\right), \varphi(t)\right) \stackrel{l \rightarrow \infty}{\longrightarrow} 0$. If $u \in[0,1]$ is a limit point of the sequence $\left(z_{k_{l}(t)}^{l}\right)_{l \in \mathbf{N}}$, from the continuity of $\varphi^{\prime}$ we should have $\varphi^{\prime}(u)=\varphi(t)$. Since $[0,1]$ is a compact set and $\left(z_{k_{l}(t)}^{l}\right)_{l \in \mathbf{N}} \subset[0,1]$ it follows that the sequence $\left(z_{k_{l}(t)}^{l}\right)_{l \in \mathbf{N}}$ has at least one limit point. If $u^{\prime} \in[0,1]$ is such that $\varphi^{\prime}\left(u^{\prime}\right)=\varphi(t)$, we should have $\varphi^{\prime}\left(u^{\prime}\right)=\varphi(t)=\varphi^{\prime}(u)$. Since $\varphi^{\prime}$ is injective, it follows that $u=u^{\prime}$. We remark that, if $u^{\prime}$ is another limit point of the sequence $\left(z_{k_{l}(t)}^{l}\right)_{l \in \mathbf{N}}$, we should have $\varphi^{\prime}\left(u^{\prime}\right)=\varphi(t)$ and so $u=u^{\prime}$. Since the sequence $\left(z_{k_{l}(t)}^{l}\right)_{l \in \mathbf{N}}$ has an unique limit point, $\left(z_{k_{l}(t)}^{l}\right)_{l \in \mathbf{N}} \subset[0,1]$ and $[0,1]$ is a compact set it follows that $z_{k_{l}(t)}^{l} \stackrel{l \rightarrow \infty}{\longrightarrow} u$.

Thus, we have proved that for every $t \in[0,1]$ there exists an unique $u(t) \in[0,1]$ such that $\varphi(t)=\varphi^{\prime}(u(t))$. This means that there exists an unique function $u:[0,1] \rightarrow[0,1]$ such that $\varphi^{\prime} \circ u=\varphi$. Since $\varphi$ is injective it results that $u$ is also injective. We will prove that $u$ is strictly increasing. 
Let $t_{1}, t_{2} \in[0,1]$ be such that $t_{1}<t_{2}$. Then there exist the sequences $\left(k_{l}\left(t_{1}\right)\right)_{l \in \mathbf{N}} \subset \mathbf{N}$ and $\left(k_{l}\left(t_{2}\right)\right)_{l \in \mathbf{N}} \subset \mathbf{N}$ such that $y_{k_{l}\left(t_{1}\right)}^{l} \leq t_{1} \leq y_{k_{l}\left(t_{1}\right)+1}^{l}$ and $y_{k_{l}\left(t_{2}\right)}^{l} \leq$ $t_{2} \leq y_{k_{l}\left(t_{2}\right)+1}^{l}$. It follows that $y_{k_{l}\left(t_{1}\right)}^{l} \leq y_{k_{l}\left(t_{2}\right)+1}^{l}$ and so $z_{k_{l}\left(t_{1}\right)}^{l} \leq z_{k_{l}\left(t_{2}\right)+1}^{l}$. Since $z_{k_{l}\left(t_{1}\right)}^{l} \stackrel{l \rightarrow \infty}{\longrightarrow} u\left(t_{1}\right), z_{k_{l}\left(t_{2}\right)}^{l} \stackrel{l \rightarrow \infty}{\longrightarrow} u\left(t_{2}\right)$ and $\left|z_{k_{l}\left(t_{2}\right)}^{l}-z_{k_{l}\left(t_{2}\right)+1}^{l}\right| \leq\left\|\Delta_{l}^{\prime}\right\| \stackrel{l \rightarrow \infty}{\longrightarrow} 0$ it follows that $u\left(t_{1}\right) \leq u\left(t_{2}\right)$.

Interchanging $\varphi^{\prime}$ with $\varphi$, there exists an unique function $v:[0,1] \rightarrow[0,1]$ such that $\varphi^{\prime}=\varphi \circ v$. Since $\varphi$ and $\varphi^{\prime}$ are injective functions, it follows that $v$ is the inverse of $u$. Therefore $u$ is bijective. Also $u$ is a continuous function, since every bijective and increasing function between two closed intervals is continuous.

Lemma 3.4. Let $(X, d)$ be a complete metric space such that $X=\bigcup_{i=1}^{n} A_{i}$, where $A_{i}$ are compact sets for $i \in\{1, \ldots, n\}$. We suppose that the associated graph of the family $\left(A_{i}\right)_{k=\overline{1, n}}$ is a tree. Let $x, y \in X, x \neq y$ a chain of sets $\left\{A_{i_{j}}\right\}_{j=\overline{0, m}}$ such that $i_{0}, i_{1}, \ldots, i_{m}$ are different, $x \in A_{i_{0}}, x \notin A_{i_{1}}, y \in A_{i_{m}}, y \notin A_{i_{m-1}}$ and $A_{i_{j}} \cap A_{i_{j+1}} \neq \emptyset$ for every $j \in\{1, \ldots, m-1\}, m \in \mathbf{N}$.

Then for any continuous, injective function $\varphi:[0,1] \rightarrow X$ such that $\varphi(0)=x$ and $\varphi(1)=y$ there exists a division $\Delta=\left(0=y_{0}<y_{1}<\cdots<y_{m}=1\right)$ of the interval $[0,1]$ such that $\varphi\left(y_{j}\right) \in A_{i_{j}} \cap A_{i_{j+1}}$ for every $j \in\{0, \ldots, m-1\}$.

Notation 3.2. Let $(X, d)$ be a complete metric space and $\mathscr{S}=\left(X,\left(f_{k}\right)_{k=\overline{1, n}}\right)$ an IFS on $X$. For every $m \in \mathbf{N}^{*}$, we denote by $\mathscr{S}^{m}$ the IFS $\mathscr{S}^{m}=\left(X,\left(f_{\omega}\right)_{\omega \in \Lambda_{m}}\right)$ and we remark that $A(\mathscr{S})=A\left(\mathscr{S}^{m}\right)$.

THEOREM 3.1. Let $(X, d)$ be a complete metric space and $\mathscr{S}=\left(X,\left(f_{k}\right)_{k=\overline{1, n}}\right)$ an IFS. We denote by $A$ the attractor of $\mathscr{S}$ and by $G^{m}$ the graph of intersections associated with the family of sets $\left(A_{\omega}\right)_{\omega \in \Lambda_{m}}$, for every $m \in \mathbf{N}^{*}$. If the graphs $G^{m}$ are trees for every $m \in \mathbf{N}^{*}$, then $A=A(\mathscr{S})$ is a dendrite.

Proof. Since $G$ is a tree it results that $G$ is connected. From theorem 1.2. it follows that $A$ is arcwise connected. We will prove that $A$ is a dendrite.

Let $x, y \in A, x \neq y$. We suppose that there exist two continuous, injective functions $\varphi, \varphi^{\prime}:[0,1] \rightarrow A$ such that $\varphi(0)=\varphi^{\prime}(0)=x$ and $\varphi(1)=\varphi^{\prime}(1)=y$. To prove that $A$ is a dendrite it is enough to prove that $\varphi$ and $\varphi^{\prime}$ are equivalent. We intend to use lemma 3.3 to prove the equivalence. For that, we will construct two sequences $\left(\Delta_{l}\right)_{l \geq 0}$ and $\left(\Delta_{l}^{\prime}\right)_{l \geq 0}$ of divisions of the unit interval $[0,1]$ such that:

a) $\Delta_{l}=\left(0=y_{0}^{l}<y_{1}^{l}<\cdots<y_{n_{l}}^{l}=1\right)$ and $\Delta_{l}^{\prime}=\left(0=z_{0}^{l}<z_{1}^{l}<\cdots<z_{n_{l}}^{l}=1\right)$ have the same number of elements for all $l \in \mathbf{N}$,

b) $\left\|\Delta_{l}\right\| \stackrel{l \rightarrow \infty}{\longrightarrow} 0$ and $\left\|\Delta_{l}^{\prime}\right\| \stackrel{l \rightarrow \infty}{\longrightarrow} 0$

c) $\max _{k=\overline{0, n_{l}}} d\left(\varphi\left(y_{k}^{l}\right), \varphi^{\prime}\left(z_{k}^{l}\right)\right) \stackrel{l \rightarrow \infty}{\longrightarrow} 0$.

Let $l \in \mathbf{N}$ be fixed. If there exists an $\alpha \in \Lambda_{l}\left(\mathbf{N}_{n}^{*}\right)$ such that $x, y \in A_{\alpha}$ then we take $\Delta_{0}=\left(y_{0}^{0}=0<y_{1}^{0}=1\right)$ and $\Delta_{0}^{\prime}=\left(z_{0}^{0}=0<z_{1}^{0}=1\right)$. We have $\varphi\left(y_{0}^{0}\right)=$ $\varphi(0)=\varphi^{\prime}(0)=\varphi^{\prime}\left(z_{0}^{0}\right)=x$ and $\varphi\left(y_{1}^{0}\right)=\varphi(1)=\varphi^{\prime}(1)=\varphi^{\prime}\left(z_{1}^{0}\right)=y$. 
If there does not exist an $\alpha \in \Lambda_{l}\left(\mathbf{N}_{n}^{*}\right)$ such that $x, y \in A_{\alpha}$, then there exists $\alpha_{x}, \alpha_{y} \in \Lambda_{l}\left(\mathbf{N}_{n}^{*}\right)$ such that $x \in A_{\alpha_{x}}, y \in A_{\alpha_{y}}$ and $\alpha_{x} \neq \alpha_{y}$.

Since $G^{l}$ is a tree, the sets $A_{\alpha_{x}}$ and $A_{\alpha_{y}}$ are joined by an unique chain of sets $\left\{A_{\omega_{j}}\right\}_{j=\overline{1, m_{l}}}$ such that $\alpha_{x}=\omega_{1}, \alpha_{y}=\omega_{m_{l}}, \omega_{j} \in \Lambda_{l}\left(\mathbf{N}_{n}^{*}\right), A_{\omega_{j}} \cap A_{\omega_{j+1}} \neq \emptyset$, for $j \in\left\{1, \ldots, m_{l}-1\right\}$ and $i_{1}, i_{2}, \ldots, i_{m_{l}}$ different. We can suppose that $x \notin A_{\omega_{2}}$ and $y \notin A_{\omega_{m_{l}-1}}$ by replacing $\alpha_{x}$ with $\omega_{2}$ if $x \in A_{\omega_{2}}$, and $\alpha_{y}$ with $\omega_{m-1}$ if $y \in A_{\omega_{m_{l}-1}}$.

From lemma 3.4. there exist $\Delta_{l}=\left(0=y_{0}^{l}<y_{1}^{l}<\cdots<y_{m_{l}}^{l}=1\right)$ and $\Delta_{l}^{\prime}=$ $\left(0=z_{0}^{l}<z_{1}^{l}<\cdots<z_{m_{l}}^{l}=1\right)$ divisions of the unit interval $[0,1]$ such that $\varphi\left(y_{j}^{l}\right), \varphi^{\prime}\left(z_{j+1}^{l}\right) \in A_{\omega_{j}} \cap A_{\omega_{j+1}}$.

It results that $\max _{k=\overline{0, m_{l}}} d\left(\varphi\left(y_{k}^{l}\right), \varphi^{\prime}\left(z_{k}^{l}\right)\right) \leq \max _{k=\overline{0, m_{l}}} d\left(A_{\omega_{k}}\right) \leq c^{l} d(A)$, where $c=\max _{k=\overline{1, n}} \operatorname{Lip}\left(f_{k}\right)<1$. Therefore $\max _{k=\overline{0, n_{l}}} d\left(\varphi\left(y_{k}^{l}\right), \varphi^{\prime}\left(z_{k}^{l}\right)\right) \stackrel{l \rightarrow \infty}{\longrightarrow} 0$.

We remark now that $d_{l}=\max _{k=\overline{0, m_{l}-1}} d\left(\varphi\left(y_{k}^{l}\right), \varphi\left(y_{k+1}^{l}\right)\right) \leq \max _{k=\overline{0, m_{l}-1}} d\left(A_{\omega_{k}}\right)$ $\leq c^{l} d(A)$. Therefore $d_{l}=\max _{k=\overline{0, m_{l}-1}} d\left(\varphi\left(y_{k}^{l}\right), \varphi\left(y_{k+1}^{l}\right)\right) \stackrel{l \rightarrow \infty}{\longrightarrow} 0$.

Let $\delta_{\mu}=\inf _{x, y \in[0,1] ;|x-y| \geq \mu} d(\varphi(x), \varphi(y))$, for every $\mu \in[0,1)$.

It is obvious that $\delta_{\mu} \leq \delta_{v}$ if $\mu \leq v$. Since $\varphi$ is injective and $[0,1]$ is a compact set, we have $\delta_{\mu}>0$ for every $\mu>0$.

We suppose by contradiction that the sequence $\left(\left\|\Delta_{l}\right\|\right)_{l \geq 0}$ is not convergent to 0 . Then there exist $\varepsilon>0$ and a subsequence $\left(\left\|\Delta_{l_{k}}\right\|\right)_{k \geq 0}$ such that $\left\|\Delta_{l_{k}}\right\| \geq \varepsilon$. Then $d_{l_{k}}=\max _{j=\overline{0, n_{l}-1}} d\left(\varphi\left(y_{j+1}^{l_{k}}\right), \varphi\left(y_{j}^{l_{k}}\right)\right) \geq \delta_{\left\|\Delta_{l_{k}}\right\|} \geq \delta_{\varepsilon}>0$. This contradicts the fact that $d_{l} \stackrel{l \rightarrow \infty}{\longrightarrow} 0$. It follows that $\left\|\Delta_{l}\right\| \stackrel{l \rightarrow \infty}{\longrightarrow} 0$. In a similar way one can prove that $\left\|\Delta_{l}^{\prime}\right\| \stackrel{l \rightarrow \infty}{\longrightarrow} 0$.

Corollary 3.1. Let $(X, d)$ be a complete metric space, $l \in \mathbf{N}^{*}$ and $\mathscr{S}=$ $\left(X,\left(f_{k}\right)_{k=\overline{1, n}}\right)$ an IFS. We denote by $A$ the attractor of $\mathscr{S}$ and by $G^{m}$ the graph of intersections associated with the family of sets $\left(A_{\omega}\right)_{\omega \in \Lambda_{m}}$, for every $m \in \mathbf{N}^{*}$. If the graph $G^{m l}$ is a tree for every $m \in \mathbf{N}^{*}$, then $A$ is a dendrite.

Proof. We can apply the theorem 3.1. to the IFS $\mathscr{S}^{l}$.

Corollary 3.2. Let $(X, d)$ be a complete metric space, $\left(n_{l}\right)_{l>1} \subset \mathbf{N}^{*} a$ strictly increasing sequence of natural numbers and $\mathscr{S}=\left(X,\left(f_{k}\right)_{k=\overline{1, n}}\right)$ an IFS. We denote by $A$ the attractor of $\mathscr{S}$ and by $G^{m}$ the graph of intersections associated with the family of sets $\left(A_{\omega}\right)_{\omega \in \Lambda_{m}}$, for every $m \in \mathbf{N}^{*}$. If the graphs $G^{n_{l}}$ are trees for every $l \in \mathbf{N}^{*}$, then $A$ is a dendrite.

Proof. Similar to the proof of theorem 3.1.

\section{Examples}

We give now some examples of attractors of IFSs which are dendrites.

Example 4.1. Let $X=\mathbf{R}$. We consider the IFS $\mathscr{S}=\left(\mathbf{R},\left(f_{k}\right)_{k=\overline{0, n-1}}\right)$, where $f_{k}: \mathbf{R} \rightarrow \mathbf{R}$ are the functions defined by $f_{k}(x)=\frac{x}{n}+\frac{k}{n}$, for $k \in\{0,1, . ., n-1\}$. 

The attractor of $\mathscr{S}$ is $A(\mathscr{S})=[0,1]$. We remark that $A_{k}=f_{k}(A)=\left[\frac{k-1}{n}, \frac{k}{n}\right]$
for $k \in\{0,1, . ., n-1\}$.

An extension of this example for countable IFSs can be found in [17], [18].

We compute the associated graphs and we obtain the following edges for every graph $G^{m}, m \in \mathbf{N}^{*}$, which prove that these are trees. Moreover, $G^{m}$ has $n^{m}-1$ edges for every $m \in \mathbf{N}^{*}$ and we have the followings edges:

$$
\begin{aligned}
& G^{1}:(0,1),(1,2), \ldots,(n-2, n-1) . \\
& G^{2}:(00,01),(01,02), \ldots,(0(n-2), 0(n-1)),(0(n-2), 10) \\
& (10,11),(11,12), \ldots,(1(n-2), 1(n-1)),(1(n-1), 20), \\
& \quad \ldots \\
& ((n-1) 0,(n-1) 1),((n-1) 1,(n-1) 2), \ldots,((n-1)(n-2),(n-1)(n-1)) . \\
& \text { In general: } \\
& G^{m}=(0 \cdots 00,0 \cdots 01),(0 \cdots 01,0 \cdots 02), \ldots,(0 \cdots 0(n-2), 0 \cdots 0(n-1)), \\
& (0 \cdots 0(n-1), 0 \cdots 010) \\
& (0 \cdots 010,0 \cdots 011),(0 \cdots 011,12), \ldots,(0 \cdots 01(n-2), 0 \cdots 01(n-1)), \\
& (0 \cdots 01(n-1), 0 \cdots 020), \\
& (0 \cdots 0(n-1) 0,0 \cdots 0(n-1) 1),(0 \cdots 0(n-1) 1,0 \cdots 0(n-1) 2), \ldots, \\
& (0 \cdots 0(n-1)(n-2), 0 \cdots 0(n-1)(n-1)) . \\
& \quad \cdots \\
& ((n-1) \cdots(n-1) 0,(n-1) \cdots(n-1) 1),((n-1) \cdots(n-1) 1, \\
& (n-1) \cdots(n-1) 2), \cdots,((n-1) \cdots(n-1)(n-2),(n-1) \cdots(n-1)(n-1)) .
\end{aligned}
$$

Notation 4.1. Let $(X, d)$ be a complete metric space and $\mathscr{S}=$ $\left(X,\left(f_{k}\right)_{k=\overline{1, n}}\right)$ an IFS. Let $\Lambda_{m}=\Lambda_{m}(\{1,2, \ldots, n\})$ the set of the words of length $m$ with the letters from the alphabet $\{1,2, \ldots, n\}$ and $\left(\Lambda_{m}, G\right)$ be a graph. By $f_{k}\left(\left(\Lambda_{m}, G\right)\right)$ we will understand the graph $\left(k \Lambda_{m}, f_{k}(G)\right)=\left(\left\{k \alpha \mid \alpha \in \Lambda_{m}\right\}\right.$, $\{(k \alpha, k \beta) \mid(\alpha, \beta) \in G\})$ for $k \in\{1,2, \ldots, n\}$.

Example 4.2. (The Hata's tree-like set).

Let $X=\mathbf{C}$. We set $f_{1}(z)=c \bar{z}$ and $f_{2}(z)=\left(1-|c|^{2}\right) \bar{z}+|c|^{2}$, where $c \in \mathbf{C}$ and $|c|,|1-c| \in(0,1)$. The attractor of the IFS formed with the functions is called a Hata's tree-like set and it is denoted by $K$. We put $A_{1}=f_{1}(K)$ and $A_{2}=f_{2}(K)$.

We compute the associated graphs and we obtain the following edges for every graph $G^{m}, m \in \mathbf{N}^{*}$, which prove that these are trees. Moreover, $G^{m}$ has $2^{m}-1$ edges for every $m \in \mathbf{N}^{*}$ and we have the followings edges:

$G^{1}:(1,2)$.

$G^{2}:(12,11),(11,21),(21,22)$.

$G^{3}:(122,121),(121,112),(112,111),(112,211),(211,212),(212,221),(221,222)$.

In general:

$G^{m}: f_{1}\left(G^{m-1}\right),(1122 \cdots 2,211 \cdots 1), f_{2}\left(G^{m-1}\right)$.

From remark 1.4 points 2) and 3) it follows that the graph $G^{m}$ is a tree for every $m$. Hence using theorem 3.1. $K$ is a dendrite. 
Example 4.3. (The Cross Set).

Let $X=\mathbf{C}$, and $A=\{z=x+i y|| x|+| y \mid \leq 1\}$. We consider the functions $f_{j}: C \rightarrow C$, where $j=0,1, \ldots, 4$, defined by $f_{0}(z)=\frac{z}{3}, f_{1}(z)=\frac{z}{3}+\frac{2}{3}, f_{2}(z)=$ $\frac{z}{3}+\frac{2 i}{3}, f_{3}(z)=\frac{z}{3}-\frac{2}{3}$ and $f_{4}(z)=\frac{z}{3}-\frac{2 i}{3}$. The attractor of the IFS formed with the functions $f_{0}, f_{1}, \ldots, f_{4}, \mathscr{S}=\left(\mathbf{C},\left\{f_{0}, f_{1}, f_{2}, f_{3}, f_{4}\right\}\right)$, is called the the cross. The fixed points of the functions $f_{0}, f_{1}, \ldots, f_{4}$, are $0,1, i,-1,-i$ and so

$0,1, i,-1,-i \in A(\mathscr{S})$.
$\quad$ We remark that $f_{0}(A)=\frac{A}{3}, f_{1}(A)=\frac{A}{3}+\frac{2}{3} \subset A, f_{2}(A)=\frac{A}{3}+\frac{2 i}{3} \subset A, f_{3}(A)=$ $\frac{A}{3}-\frac{2}{3} \subset A$ and $f_{4}(A)=\frac{A}{3}-\frac{2 i}{3} \subset A$. Therefore $F_{\mathscr{S}}(A) \subset A$ and $A(\mathscr{S}) \subset A$. Also $f_{1}(A(\mathscr{S})) \cap f_{3}(A(\mathscr{S})) \subset\left(\frac{A}{3}+\frac{2}{3}\right) \cap\left(\frac{A}{3}-\frac{2}{3}\right)=\emptyset$.

In a similar way one can obtain that $f_{l}(A(\mathscr{S})) \cap f_{j}(A(\mathscr{S}))=\emptyset$, for every $l, j \in\{1,2,3,4\}$ such that $l \neq j$.

We also remark that on one side we have $f_{0}(A(\mathscr{S})) \cap f_{1}(A(\mathscr{S})) \subset \frac{A}{3} \cap$ $\left(\frac{A}{3}+\frac{2}{3}\right)=\left\{\frac{1}{3}\right\}$ and on the other side $\frac{1}{3}=f_{0}(1)=f_{1}(-1)$. Thus $f_{0}(A(\mathscr{S})) \cap$ $f_{1}(A(\mathscr{S}))=\left\{\frac{1}{3}\right\}$. In a similar way one can obtain that $f_{0}(A(\mathscr{S})) \cap f_{2}(A(\mathscr{S}))=$ $\left\{\frac{2 i}{3}\right\}, f_{0}(A(\mathscr{S})) \cap f_{3}(A(\mathscr{S}))=\left\{-\frac{2}{3}\right\}$ and $f_{0}(A(\mathscr{S})) \cap f_{4}(A(\mathscr{S}))=\left\{-\frac{2 i}{3}\right\}$.

We compute the associated graphs and we obtain the following edges for every graph $G^{m}, m \in \mathbf{N}^{*}$, which prove that these are trees. Moreover, $G^{m}$ has $5^{m}-1$ edges for every $m \in \mathbf{N}^{*}$ and are described below:

$G^{1}:(0,1),(0,2),(0,3),(0,4)$.

$G^{2}:(00,01),(00,02),(00,03),(00,04)$,

$(01,13),(13,10),(10,11),(10,12),(10,14)$,

$(02,24),(24,20),(20,21),(20,22),(20,23)$,

$(03,31),(31,30),(30,32),(30,33),(30,34)$,

$(04,42),(42,40),(40,41),(40,43),(40,44)$.

In general:

$G^{m}: f_{0}\left(G^{m-1}\right),(011 \cdots 1,133 \cdots 3), f_{1}\left(G^{m-1}\right),(022 \cdots 2,244 \cdots 4)$,

$f_{2}\left(G^{m-1}\right),(033 \cdots 3,311 \cdots 1), f_{3}\left(G^{m-1}\right),(022 \cdots 2,244 \cdots 4), f_{4}\left(G^{m-1}\right)$,

From remark 1.4 points 2) and 3$)$ it follows that the graph $G^{m}$ is a tree for every $m$. Hence using theorem 3.1 . the cross set is a dendrite.

\section{REFERENCES}

[1] J. Andres, J. Fišer, G. Gabor and K. LeŚniak, Multivalued fractal, Chaos, Solitons and Fractals 24 (2005), 665-700.

[2] M. F. Barnsley, Fractals everywhere, Academic Press, New York, 1998. 
[ 3 ] D. Dumitru and A. Minail, A sufficient condition for the connectedness of the attractors of infinite iterated function systems, An. Ştiinţ. Univ. Al. I. Cuza Iaşi, Mat. (N.S.) 55 (2009), 87-94.

[4] K. J. Falconer, The geometry of fractal sets, Cambridge Univ. Press, 1985.

[5] K. J. FALCONER, Fractal geometry-foundations and applications, John Wiley, 1990.

[6] J. Hutchinson, Fractals and self-similarity, Indiana Univ. J. Math. 30 (1981), 713-747.

[7] J. Kigami, Analysis on fractals, Cambridge univ. Press, 2001.

[ 8 ] K. LeŚNIAK, Infinite iterated function systems: A multivalued approach, Bull. Pol. Ac. Math. 52 (2004), 1-8.

[9] R. Miculescu AND A. Mihail, Lipscomb's space $\varpi^{A}$ is the attractor of an IFS containing affine transformation of $l^{2}(A)$, Proceedings of AMS 136 (2008), 587-592.

[10] R. Miculescu and A. Mihail, Applications of fixed point theorems in the theory of generalized IFS, Fixed Point Theory and Appl. 2008, Article ID 312876.

[11] R. Miculescu and A. Mihail, The shift space for an infinite iterated function system, Math. Reports 1 (2009), 21-32.

[12] R. Miculescu and A. Mihail, Generalized IFSs on noncompact spaces, Fixed Point Theory and Appl. 2010, Article ID 584215.

[13] R. Miculescu and A. Mihail, The Hutchinson measure for generalized iterated function systems, Rev. Roumaine Math. Pures Appl. 54 (2009), 297-316.

[14] A. MiHAIL, On the connectivity of attractors of iterated multifunction systems, Real Analysis Exchange 34 (2008), 195-206.

[15] N. A. Secelean, Countable iterated function systems, Far East J. Dyn. Syst. 3 (2001), 149167.

[16] N. A. Secelean, Măsură şi Fractali, Ed. Lucian Blaga, Univ. Sibiu, 2002.

[17] N. A. SEcelean, Parametrized curve as attractors of some countable iterated function systems, Arch. Math. Brno 40 (2004), 287-293.

[18] N. A. Secelean, The fractal interpolation for countable system data, Univ. Beograd. Publ. Elektrotehn. Fak., Ser. Mat. 14 (2003), 11-19.

[19] N. A. Secelean, Any compact subset of a metric space is the attractor of a countable function system, Bull. Math. Soc. Sci. Math. Roumanie 44 (2001), 237-241.

[20] M. Yamaguchi, M. Hata and J. Kigami, Mathematics of fractals, Translations of mathematical monographs 167, AMS, 1997.

Dan Dumitru

"SPiru HaReT" UNIVERSity OF Bucharest

Department of Mathematics and Computer Science

13, Ghica Str., Bucharest

ROMANIA

E-mail: dandumitru1984@yahoo.com

\author{
Alexandru Mihail \\ UNIVERSITY OF BUCHAREST \\ Department of Mathematics and Computer Science \\ 14, Academiei Str., Bucharest \\ ROMANIA \\ E-mail: mihail_alex@yahoo.com
}

